\title{
Inventario de Ansiedad de Beck: validez estructural y fiabilidad a través de distintos métodos de estimación en población argentina
}

\author{
Nicolás Alejandro Vizioli; Alejandro Emilio Pagano
}

Cómo citar este artículo:

Vizioli, N., A., \& Pagano, A., E. (2022). Inventario de Ansiedad de Beck: validez estructural y fiabilidad a través de distintos métodos de estimación en población argentina. Acta Colombiana de Psicología, 25(1), 28-41. https://www.doi. org/10.14718/ACP.2022.25.1.3

Recibido, febrero 26/2021; Concepto de evaluación, junio 14/2021; Aceptado, septiembre 16/2021

\author{
Nicolás Alejandro Vizioli ${ }^{1}$ \\ ORCID: https://orcid.org/0000-0002-6113-6847 \\ Universidad de Buenos Aires, Ciudad Autónoma de Buenos Aires, Argentina. \\ Alejandro Emilio Pagano \\ ORCID: https://orcid.org/0000-0003-4817-9145 \\ Universidad de Buenos Aires, Ciudad Autónoma de Buenos Aires, Argentina.
}

Resumen

\begin{abstract}
La validez y fiabilidad como propiedades psicométricas consisten en complejos procesos que deben ser revisados constantemente para garantizar la correcta utilización de un instrumento de medición. Por tanto, en el presente estudio se indagó respecto a la validez estructural, la validez factorial y la fiabilidad del Inventario de Ansiedad de Beck (BAI) en su adaptación argentina, a partir de diferentes métodos en una muestra de 746 participantes $\left(M_{\text {edad }}=33.49, D E=10.55\right)$ pertenecientes a la ciudad de Buenos Aires y al conurbano Bonaerense, en Argentina. Específicamente, se realizaron análisis factoriales confirmatorios para probar los modelos de una, dos y cuatro dimensiones, utilizando los métodos de estimación de mínimos cuadrados no ponderados, máxima verosimilitud robusto, mínimos cuadrados ponderados diagonales, y mínimos cuadrados generalizados robusto. Adicional a esto, se indagó respecto a la fiabilidad de la prueba mediante el alfa, omega, GLB algebraico y factorial, $\mathrm{H}$, beta, y theta. Los resultados indican que el modelo unidimensional mostró mejores índices de bondad de ajuste independientemente del método de estimación; y que todos los índices de fiabilidad obtenidos fueron aceptables. Esta investigación aporta sólidas evidencias sobre la validez estructural y la fiabilidad del BAI.

Palabras clave: validez, fiabilidad, propiedades psicométricas, BAI.
\end{abstract}

\section{Beck Anxiety Inventory: structural validity and reliability through different estimation methods in Argentine population}

\begin{abstract}
Validity and reliability as psychometric properties consist of complex processes that must be constantly reviewed to ensure the correct use of a measurement instrument. Therefore, the present study investigated the structural validity, factorial validity, and reliability of the Beck Anxiety Inventory (BAI) in its Argentine adaptation, using different methods in a sample of 746 participants (Mean $=33.49, \mathrm{SD}=10.55$ ) belonging to the city of Buenos Aires and the Buenos Aires suburbs, in Argentina. Specifically, confirmatory factor analyses were performed to test the one-, two- and four-dimensional models, using unweighted least squares, robust maximum likelihood, diagonal weighted least squares, and robust generalized least
\end{abstract}

1 Facultad de Psicología, Universidad de Buenos Aires, Ciudad Autónoma de Buenos Aires, Argentina. nicovizioli@gmail.com 
squares estimation methods. In addition, test-retest reliability was investigated using alpha, omega, algebraic and factorial GLB, H, beta, and theta. The results indicate that the unidimensional model showed better goodness-of-fit indices regardless of the estimation method; and that all the reliability indices obtained were acceptable. This research provides strong evidence on the structural validity and reliability of the BAI.

Keywords: validity, reliability, psychometric properties, BAI.

\section{Introducción}

El Inventario de Ansiedad de Beck (BAI), desarrollado originalmente por Beck et al. (1988), es el instrumento de evaluación más citado en las bases de datos científicas (Piotrowski, 2018), posiblemente debido a que los trastornos de ansiedad son los de mayor prevalencia a nivel mundial (Datani et al., 2018). Dada su popularidad, distintos estudios se han centrado en analizar las propiedades psicométricas de este instrumento.

En cuanto su estructura factorial, inicialmente, Beck et al. (1988) obtuvieron 21 ítems resultantes de un conjunto de 86 ítems que conformarían la versión final del BAI; y a partir de ellos hallaron una estructura de dos factores, uno que hace referencias a síntomas somáticos y otro que mide sintomatología asociada a síntomas subjetivos de ansiedad y pánico. En cuanto a su consistencia interna, informaron un alfa de .92 (Beck \& Steer, 1993).

Posteriormente, como se encuentra en un metaanálisis realizado por Bardhoshi et al. (2016) sobre las propiedades psicométricas de las versiones de habla inglesa del BAI, se encontraron 17 investigaciones que realizaban análisis factoriales exploratorios ( $\mathrm{AFE}$ ), en las cuales se sugirieron estructuras de entre uno y seis factores para la prueba: en 11 de estos estudios se evidenció una adecuación de soluciones de dos factores, en cuatro estudios se sugirió la adecuación de modelos de cuatro factores, una investigación informó una solución de cinco factores, y otra propuso una solución de seis factores. Asimismo, en cuanto a análisis factoriales confirmatorios (AFC), Bardhoshi et al. (2016) informaron de ocho estudios que aplicaron AFC para probar varios modelos de BAI: cinco probaron un modelo unidimensional - aunque solo una halló que los datos mostraban un ajuste adecuado-, y la mayoría encontró que el modelo original de dos factores ofrecía índices de bondad de ajuste adecuados, mientras que las pruebas de modelos de cuatro, cinco y seis factores mostraron valores inadecuados de ajuste. Respecto a la fiabilidad, Bardhoshi et al. (2016) encontraron 117 estudios que reportan la consistencia del instrumento a través del alfa, y 18 que examinan la estabilidad temporal — con una mediana de seis semanas de intervalo-; en cuanto a los coeficientes alfa, los puntajes oscilaron entre .81 y .95 , y en los otros casos se reportó una estabilidad temporal de .65 en promedio.

Adicionalmente, en los últimos años se han llevado a cabo distintas investigaciones a nivel internacional respecto a la estructura y las propiedades psicométricas del BAI en distintos tipos de población. Por ejemplo, Benuto et al. (2020) reportaron el ajuste de un modelo de dos factores en una muestra de 149 latinos hispanohablantes pacientes de dispositivos de salud primaria estadounidenses; Chapa (2021), por su parte, halló la adecuación de un modelo de cuatro factores en una muestra de 416 trabajadores estadounidenses; Liang et al. (2018) reportaron el ajuste de un modelo de cuatro factores y un alfa de .94 en una muestra de 762 médicos chinos; Clark et al. (2016) encontraron que, en 208 pacientes cardíacos norteamericanos en rehabilitación, los mejores valores de ajuste se hallaban en una estructura con una dimensión global y cuatro factores de segundo orden; Palmer et al. (2016), en Estados Unidos, concluyeron que el modelo de cinco factores exhibía mejor ajuste en una muestra de 304 militares retirados que habían sufrido politraumatismos; Lee et al. (2018) informaron un modelo de cinco factores en una muestra de 406 pacientes psiquiátricos coreanos; y, por último, Adhikari (2019) encontró, en 2007 adolescentes de Nepal, índices adecuados de validez concurrentes y un alfa de .86 con una estructura de seis factores.

En cuanto a investigaciones realizadas en español, Sanz y Navarro (2003) hallaron, a través de distintos AFE en 590 estudiantes universitarios españoles, una consistencia interna con un alfa de .88 y una solución factorial de una dimensión de ansiedad compuesta por dos factores interrelacionados, correspondientes a síntomas somáticos y afectivo-cognitivos; Sanz et al. (2012) corroboraron, mediante un AFE, la estructura de una dimensión del BAI 
y hallaron un alfa de .90 en una muestra de 307 pacientes españoles con trastornos psicológicos; Magán et al. (2008) informaron en 249 adultos españoles un alfa de .85 y una estructura compuesta por una dimensión global de ansiedad con un factor somático y uno afectivo; Robles et al. (2001) adaptaron el BAI a población mexicana adulta e informaron una alfa de .83 y una estructura factorial de cuatro componentes principales; y Guillén Díaz-Barriga y González-Celis Rangel (2019) reportaron un alfa de .89 y confirmaron la estructura de cuatro factores a través de un análisis factorial exploratorio en una muestra de 157 pacientes asmáticos mexicanos.

Por su parte, Padrós Blázquez et al. (2020) realizaron un AFC mediante el cual hallaron un alfa de .91, y probaron modelos unidimensionales y de cuatro factores, aunque no obtuvieron valores de ajuste aceptables en ninguno de los dos; Rodríguez Reynaldo et al. (2001) estudiaron las propiedades psicométricas del BAI en una muestra de 150 adultos mayores portorriqueños cuyas edades oscilaron entre los 60 y los 99 años, y mediante un AFE hallaron la adecuación de una solución de tres factores y un alfa de .94; y, por último, Vizioli y Pagano (2020) hallaron en una muestra de 269 adultos argentinos que el modelo unidimensional evidenció mejores índices de ajuste que la solución bifactorial y la de cuatro factores - aunque las diferencias en los índices de bondad de ajuste de los modelos de una y cuatro dimensiones fue mínima-, y en cuanto a la fiabilidad, informaron un omega ordinal de .95, un alfa ordinal de .93 y un coeficiente rho de .92 .

Como se puede observar, las investigaciones muestran que no existe un acuerdo acerca de la estructura factorial del BAI, lo cual da cuenta de la necesidad de continuar con la investigación en las mismas, inclusive a través de distintos métodos de estimación. También, resalta que la mayoría de los estudios estimaron la consistencia interna a través del coeficiente alfa, cuya adecuación para instrumentos con formato de respuesta ordinal — como el BAI — es relativa. Y por último, es de destacar que las investigaciones sobre el BAI en Argentina son aún incipientes.

\section{Análisis factoriales confirmatorios y métodos de estimación para datos ordinales}

Los análisis factoriales confirmatorios son una de las aplicaciones de los modelos de ecuaciones estructurales (Hoyle, 2012) y, por ello, permiten especificar y evaluar un modelo, así como comparar distintos modelos, y desarrollar un modelo mediante modificaciones (Cupani, 2012; Kline, 2011), lo cual suele realizarse a partir de seis pasos: especificación, identificación, estimación de parámetros, evaluación del ajuste, reespecificación del modelo, e interpretación de resultados (Cupani, 2012).

En la fase de estimación de parámetros es posible usar distintos métodos de acuerdo con las características de los datos. Ahora bien, la calidad de las estimaciones de los parámetros del modelo, del error y de los índices de bondad de ajuste dependen de la elección del método de estimación (Lei \& Wu, 2012), ya que pueden existir diferencias en función de la posible influencia del método utilizado (Kogar \& Yilmaz-Kogar, 2015). De hecho, la estimación de parámetros a partir de matrices de correlaciones policóricas ha mostrado resultados robustos (Coenders et al., 1997; Flora \& Curran, 2004), por lo que Yang-Wallentin et al. (2010) proponen el uso de métodos robustos, ya que estiman matrices de covarianza asintóticas a partir de matrices de correlaciones policóricas.

Sin embargo, las recomendaciones acerca de qué método utilizar varían. Por ejemplo, Holgado-Tello et al. (2018) recomiendan el uso de mínimos cuadrados no ponderados robustos (RULS); Raykov y Marcoulides (2006), el uso del método máxima verosimilitud robusto (RML) con muestras pequeñas o moderadas, y el de mínimos cuadrados ponderados (WLS) con muestras suficientemente grandes; y DiStefano et al. (2019), el método de mínimos cuadrados con ponderación diagonal (DWLS).

En los últimos años, se han realizado distintas investigaciones que comparan métodos de estimación con datos ordinales. Por ejemplo, Savalei y Rhemtulla (2013) compararon los métodos RULS y DWLS mediante una simulación de Monte Carlo, y hallaron que el RULS rindió mejor que el DWLS en términos de error de Tipo 1, ya que el DWLS tendía a sobreestimarlo, mientras que el RULS rindió mejor que el DWLS cuando las muestras eran pequeñas o moderadas, y que ambos métodos ofrecían un rendimiento pobre en situaciones donde había muestras pequeñas, pocas categorías $\mathrm{y}$ valores extremos.

Por otro lado, Bandalos (2014) comparó los métodos de máxima verosimilitud (ML), RML, DWLS y WLS a través de distintas condiciones, y reportó que el método de DWLS presentó menor sesgo en la estimación de parámetros y de errores estándar en la mayoría de las condiciones, que el 
RML también tuvo un rendimiento aceptable en términos de sesgos al estimar parámetros y errores estándar, y que tanto el DWLS como el RML rindieron de una manera menos adecuada en condiciones caracterizadas por alta asimetría, tamaños de muestra pequeños y especificación incorrecta del modelo. En cuanto al WLS, este método resultó tener mayor sesgo en todas las condiciones.

Asimismo, Kogar y Yilmaz-Kogar (2015) compararon los métodos ML, ULS y DWLS en una muestra de 548 personas asignadas aleatoriamente a dos grupos - uno de $250 \mathrm{y}$ otro de 500-, y hallaron que los índices de bondad de ajuste fueron mayores en el caso de DWLS, seguido de ULS y de ML.

También, Li (2016a) comparó los métodos RML y DWLS mediante una simulación de Monte Carlo, y encontró que el DLWS mostró menos sesgos y mayor precisión que el RML al estimar las cargas factoriales en casi todas las condiciones, aunque el DWLS sobreestimó las correlaciones entre factores cuando los tamaños muestrales eran pequeños y cuando las distribuciones eran moderadamente no normales, y el método RML rindió mejor que el DWLS en cuanto a las estimaciones del error de las cargas factoriales en casos donde las distribuciones latentes no eran normales con un tamaño de muestra pequeño - menor a 200-.

Incluso, Li (2016b), mediante una simulación de Monte Carlo, comparó los métodos RML, DWLS y RULS a través de 84 condiciones caracterizadas por diferentes formas de distribución ordinales observadas, categorías de respuesta y tamaños muestrales, y halló que los métodos DWLS y RULS produjeron estimaciones de cargas factoriales, correlaciones y estimaciones de coeficientes más precisas que el RML en todas las condiciones, aunque los errores estándar de las estimaciones de los parámetros obtenidos con RML fueron más precisos, y el RML fue inferior al DWLS y el ULS en el control del error de Tipo I, excepto en muestras mayores a 1000 .

Adicionalmente, Holgado-Tello et al. (2018) analizaron los cambios en chi cuadrado obtenidos al comparar los métodos RML, WLS, ULS y RULS, y hallaron que el método RULS redujo la probabilidad de rechazar modelos con especificaciones correctas y aumentó la probabilidad de rechazar modelos mal especificados, y que el RULs mostró menor probabilidad de error de Tipo 1 que los métodos ML y wLS, pero mayor que el RML bajo ciertas condiciones.

Por último, Nalbantoğlu-Yılmaz (2019) comparó los resultados obtenidos mediante los métodos ML, RML y WLS a través de simulaciones de Monte Carlo con muestras que no cumplían los supuestos de normalidad, y encontró que las mejores estimaciones con muestras pequeñas — de 300 o 500 participantes - fueron con el método RML, mientras que con muestras con más de 1000 participantes el WLS fue superior.

Como se puede observar, la elección de un método de estimación es un punto controvertido, por lo que se ha sugerido utilizar varios métodos de estimación en casos donde no se cumplan los supuestos de normalidad (NalbantoğluYllmaz, 2019). No obstante, las investigaciones sugieren que los métodos RULS, RML y DWLS son adecuados cuando se cuenta con datos ordinales y muestras no muy grandes, mientras que el WLS puede ser de utilidad para muestras grandes.

\section{El alfa y coeficientes alternativos para estimar la fiabilidad}

Otro punto de conflicto en la realización de estudios psicométricos es la fiabilidad, dado que existen distintos coeficientes que permiten estimarla. Entre ellos, por lo general, se encuentran el alfa (Cronbach, 1951), la beta (Revelle, 1979), los omega total, jerárquico y asintótico (McDonald, 1999), el H (Hancock \& Mueller, 2001), la theta (Armor, 1973), y el de mayores límites inferiores (greatest lower bound, GBL, Jackson \& Agunwamba, 1977), pero el más utilizado históricamente ha sido el coeficiente alfa, y su popularidad se debe, en parte, a su fácil cálculo, su facilidad para comunicar, la posibilidad de calcularlo mediante una sola aplicación de una prueba, y su presencia en distintos softwares estadísticos (Viladrich et al., 2017); incluso, se ha propuesto un alfa para datos ordinales (Oliden \& Zumbo, 2008; Zumbo et al., 2007), que se calcula a partir de matrices de correlaciones policóricas con la finalidad de evitar posibles infraestimaciones (Gadermann et al., 2012).

No obstante, existen distintos argumentos por los cuales se ha desaconsejado el uso del coeficiente alfa en distintas situaciones, como que se trata de un coeficiente afectado por el número de ítems que miden un constructo, o que el cálculo del alfa exige el cumplimiento de supuestos que difícilmente se sostienen en situaciones con datos reales - la tau-equivalencia, la unidimensionalidad y la ausencia de errores correlacionados-(McNeish, 2018; Raykov, 1998). Sin embargo, es preciso destacar que, si cumple con estos supuestos, es el coeficiente recomendado y tiene utilidad (Raykov \& Marcoulides, 2019). A su vez, en ausencia de 
tau-equivalencia, el coeficiente alfa funciona bien como mayor límite inferior (Cronbach, 1951; Trizano-Hermosilla \& Alvarado, 2016), lo que quiere decir que permite estimar la mayor fiabilidad posible en ausencia del cumplimiento de los supuestos (Sijtsma, 2009).

Otro de los métodos recomendados para calcular la fiabilidad es el coeficiente GLB (Sijtsma, 2009), pero, dada la dificultad para calcularlo, se han propuesto dos procedimientos: el cálculo mediante el análisis factorial de rangos mínimos (Trizano-Hermosilla \& Alvarado, 2016) y el llamado GLB algebraico (Moltner \& Revelle, 2015).

Adicionalmente, según Revelle y Zinbarg (2009), un estimador alternativo que se puede utilizar como mayor límite inferior es el coeficiente omega, un coeficiente apto para modelos congenéricos, que se calcula a través de cargas factoriales, y cuyos valores suelen ser ligeramente más elevados que los obtenidos mediante el coeficiente alfa (Revelle \& Zinbarg, 2009). En casos donde los datos son unidimensionales, el omega total y el omega jerárquico son equivalentes (Trizano-Hermosilla \& Alvarado, 2016).

Asimismo, Revelle y Zinbarg (2009) han apuntado la importancia del cálculo del coeficiente beta como forma de estimar la fiabilidad de la peor mitad de un instrumento, debido a que utiliza el análisis de conglomerados jerárquico para hallar la peor mitad del instrumento y calcular su fiabilidad.

Una alternativa adicional es el cálculo del coeficiente theta, que se calcula a partir del análisis de componentes principales con el objetivo de estimar la varianza de las variables observables (Domínguez-Lara, 2012; Elosua \& Zumbo, 2008).

Y por último, se ha planteado el coeficiente $H$ como una medida complementaria que permite la estimación de la fiabilidad del constructo, que puede interpretarse como el porcentaje de la variabilidad de la variable latente explicada por los indicadores (Domínguez-Lara, 2016; Hancock \& Mueller, 2001).

Dada la utilidad de cada índice de fiabilidad, sería adecuado reportar distintos índices sobre una misma muestra. Por esta razón, en el presente trabajo se propone obtener evidencias sobre la validez estructural y la fiabilidad de la versión argentina del BAI, a partir de la comparación de los modelos de uno, dos y cuatro factores, mediante distintos métodos de estimación. Específicamente, se hizo uso los métodos RULS, RML y DWLS, cuya adecuación para datos ordinales ha sido demostrada en distintas investigaciones, y se realizó la estimación mediante el método de mínimos cuadrados generalizados robusto (RGLS), del cual no se han hallado investigaciones con datos ordinales - no se computaron cálculos con el método wLS, dado que no se pudo obtener una muestra suficientemente grande-; y en cuanto a la fiabilidad, se computaron los índices alfa, omega, GLB algebraico y factorial, la beta, la $\mathrm{H}$ y la theta.

\section{Método}

\section{Tipo de estudio}

Se realizó un estudio instrumental (Montero \& León, 2002), mediante el cual se analizaron las propiedades psicométricas del Inventario de Ansiedad de Beck (BAI).

\section{Participantes}

Mediante un muestreo intencional no probabilístico, se recogió una muestra de 746 participantes, con edades entre los 18 y los 65 años $(M=33.49 ; D E=10.55) .485(65 \%)$ de los participantes residían en el conurbano Bonaerense y 261 ( $35 \%$ ) en la ciudad de Buenos Aires, Argentina; en cuanto al género, 538 (72\%) informaron género femenino y 208 (28\%), masculino; y con respecto al nivel de instrucción, 239 (32\%) reportaron estudio universitario incompleto, $166(22 \%)$ universitario completo, $116(15.5 \%)$ secundario completo, $78(10.5 \%)$ terciario completo, $59(8 \%)$ terciario incompleto, 48 (6\%) posgrado, $35(5 \%)$ secundario incompleto, y 5 (1\%) primario completo.

\section{Instrumentos}

\section{Cuestionario sociodemográfico}

Se elaboró un cuestionario sociodemográfico específico para este estudio, que buscaba indagar sobre el lugar de residencia, la edad, el género y el nivel educativo de los participantes.

\section{Inventario de Ansiedad de Beck}

Se hizo uso de la adaptación argentina de Vizioli y Pagano (2020) del Inventario de Ansiedad de Beck (BAI) (Beck et al., 1988), un instrumento autoadministrable de 21 ítems que mide síntomas característicos de la ansiedad. 
La puntuación de los reactivos se realiza sobre una escala Likert de 4 opciones —que van de 0 ("Para nada") a 3 ("Severamente-Me molestó mucho")_. El inventario presenta propiedades psicométricas adecuadas para la población argentina, con evidencias de validez de constructo a través de análisis factoriales confirmatorios, y una consistencia interna de alfa ordinal de .93 , y un omega ordinal de .95 (Vizioli \& Pagano, 2020).

Aunque el estudio de validación original de Beck et al. (1988) planteaba una estructura bifactorial mediante análisis factoriales exploratorios, Vizioli y Pagano (2020), al comparar estructuras de uno, dos y cuatro factores, hallaron la adecuación de un modelo unifactorial a través de distintos AFC en población argentina; hallazgo que concuerda con lo registrado en población española (Magán et al., 2008; Sanz \& Navarro, 2003; Sanz et al., 2012).

\section{Procedimiento}

La recolección de datos se realizó bajo responsabilidad de los autores, mediante plataformas virtuales en las que se incluyó el cuestionario sociodemográfico y el BAI. Adicional a esto, los participantes debían completar un consentimiento informado en el cual se especificaban los detalles de los objetivos y los lineamientos generales de la investigación, las garantías de anonimato, que los participantes podían abandonar el estudio en el momento en que lo consideraran necesario, que la participación era voluntaria y sin compensación, y que, en caso de sentir malestar o presentar disconformidad con la investigación, podían comunicarse con responsable de esta, con garantía de la confidencialidad de la comunicación. Los participantes dieron su consentimiento mediante la selección de una de dos opciones de respuesta al comienzo de la administración: "Deseo participar" o "No deseo participar". En total, la administración de los instrumentos tomó entre 15 y 20 minutos.

\section{Aspectos éticos}

En todos los casos se respetó el resguardo del anonimato y se obtuvo el consentimiento informado de los participantes que conformaron la muestra. Antes de presentar los instrumentos, se explicó el objetivo de la investigación y se informó a los participantes que podían finalizar su participación cuando quisieran, así como el carácter anónimo y confidencial de los datos.
Finalmente, es importante mencionar que la presente investigación se ciñe a la Declaración de Helsinki (World Medical Association, 2013), en la cual se establecen los principios éticos fundamentales para la investigación con seres humanos.

\section{Análisis de datos}

Para obtener las evidencias de validez estructural del BAI, se realizaron distintos AFC, y, dada la naturaleza ordinal de las opciones de respuesta, se trabajó sobre matrices de correlaciones policóricas (Jöreskog \& Sörbom, 1996).

Como paso previo, se analizó la normalidad multivalente de las variables estudiadas, siguiendo las recomendaciones de Morata-Ramírez et al. (2015). Dados los elevados niveles de asimetría y curtosis, se procedió a utilizar métodos robustos (Freiberg Hoffmann et al., 2020; Holgado-Tello et al., 2018), y posteriormente se realizaron estimaciones mediante los métodos RULS, RML, DWLS y RGLS de los modelos de un factor, dos factores y cuatro factores. Los cálculos se realizaron con el software EQS 6.1, con excepción del método DWLS, que se computó con el paquete lavaan (Rosseel, 2012) del programa R.

Específicamente, se utilizaron cuatro índices de bondad de ajuste robustos: (a) el $x^{2}$ de Satorra-Bentler (S-B $x^{2}$ ) dividido por los grados de libertad - valores $\leq 5.0$ indican un buen ajuste-; (b) el NNFI (Non-Normed Fit Index); (c) el CFI (Comparative Fit Index); y (d) el RMSEA (Root Mean Square Error of Approximation). De acuerdo con los criterios especificados por Kline (2011) y Schumacker y Lomax (2016), se consideró un ajuste aceptable a valores mayores o iguales a .90 en NNFI y CFI. Respecto al RMSEA, valores menores a .05 indican un buen ajuste, y valores entre .05 y .08, un ajuste razonable (Browne \& Cudeck, 1993). Para la validez de constructo, se consideraron aceptables cargas estandarizadas mayores a .40 (Brown, 2006).

Antes del cálculo de la fiabilidad, se evaluó el ajuste del modelo tau-equivalente con el fin de determinar la adecuación del cálculo del coeficiente alfa (Dunn et al., 2014). Este modelo asume igualdad estadística de cargas factoriales, por lo que se restringieron las cargas factoriales a fin de probar el modelo.

Luego, se calcularon distintos índices a partir de la matriz de correlaciones policóricas: el alfa, el omega, el GLB algebraico y factorial, el $\mathrm{H}$, la beta y la theta. Los coeficientes alfa, omega, GLB y beta se calcularon mediante 
el software R, versión 3.6.0, con los paquetes GPArotation (Bernaards \& Jennrich, 2005), psych (Revelle, 2018) y Rcmdr (Fox et al., 2019); el coeficiente H se calculó con el software EQS, versión 6.1; y la theta se calculó con el módulo de Excel facilitado por Domínguez-Lara (2018). Siguiendo a Nunnally y Bernstein (1994), se consideraron aceptables valores superiores a .80 en todos los coeficientes (Henseler et al., 2009).

Por último, se calculó el intervalo de confianza del $95 \%$ del coeficiente alfa mediante la herramienta propuesta por Domínguez-Lara y Merino-Soto (2015). Complementariamente, se calcularon las correlaciones corregidas ítem-factor, considerando como adecuados los valores superiores a .40 (Nunnally \& Bernstein, 1994).

\section{Resultados}

Respecto a las comparaciones entre modelos, los índices de bondad de ajuste indican que el modelo unidimensional ofreció mejores índices de bondad de ajuste, independientemente del método de estimación utilizado (véase Tabla 1). Como se puede observar, el modelo unidimensional obtuvo valores aceptables con todos los métodos de estimación utilizados; incluso, los índices de bondad de ajuste resultaron comparativamente mejores para el modelo unidimensional en cada método de estimación.

Tabla 1.

Índices de bondad de ajuste de los modelos de 1, 2 y 4 factores del BAI mediante distintos métodos de estimación

\begin{tabular}{ccccccc}
\hline Modelo & Método & $x^{2}$ & GL & NNFI & CFI & $\begin{array}{c}\text { RMSEA } \\
\text { (IC 90 \%) }\end{array}$ \\
\hline \multirow{5}{*}{1 factor } & RULS & 930712 & 189 & .95 & .96 & $.07(.07-.08)$ \\
& RML & 1041.852 & 189 & .94 & .95 & $.08(.07-.08)$ \\
& DWLS & 947305 & 189 & .98 & .98 & $.07(.07-.08)$ \\
& RGLS & 263414 & 189 & .99 & .99 & $.02(.01-.03)$ \\
\hline \multirow{5}{*}{ factores } & RULS & 8183.176 & 189 & .57 & .61 & $.21(.21-.22)$ \\
& RML & 1085.383 & 189 & .94 & .95 & $.08(.08-.08)$ \\
& DWLS & 1073.790 & 189 & .93 & .93 & $.08(.07-.08)$ \\
& RGLS & 1531.215 & 189 & .73 & .76 & $.10(.09-.10)$ \\
\hline \multirow{5}{*}{4 factores } & RULS & 10849.334 & 189 & .62 & .67 & $.20(.19-.20)$ \\
& RML & 12140.699 & 189 & .93 & .94 & $.09(.08-.09)$ \\
& DWLS & 12801.17 & 189 & .94 & .95 & $.07(.06-.07)$ \\
& RGLS & 1585.482 & 189 & .72 & .75 & $.10(.09-.10)$ \\
\hline
\end{tabular}

Teniendo en cuenta que el modelo unidimensional ofrece mejores índices de ajuste, en la Tabla 2 se presentan estadísticos descriptivos y correlaciones corregidas ítem-factor de los ítems del BAI. Como se puede observar, las correlaciones corregidas ítem-factor resultaron adecuadas en todos los casos, con valores que oscilaron entre .50 y .77.

Tabla 2.

Estadísticos descriptivos y correlaciones corregidas item-factor de los reactivos del BAI

\begin{tabular}{|c|c|c|c|}
\hline Ítems & $M$ & $D E$ & $\begin{array}{l}\text { Correlación } \\
\text { corregida } \\
\text { item-factor }\end{array}$ \\
\hline 1. Hormigueo o entumecimiento. & 1.6 & 0.76 & .50 \\
\hline 2. Sensación de calor intenso. & 1.6 & 0.79 & .51 \\
\hline 3. Debilidad en las piernas. & 1.5 & 0.76 & .59 \\
\hline 4. Incapacidad para relajarse. & 2.4 & 0.96 & .69 \\
\hline 5. Miedo a que pase lo peor. & 2.1 & 1.04 & .72 \\
\hline 6. Mareos o vértigos. & 1.4 & 0.70 & .68 \\
\hline 7. Palpitaciones o taquicardia. & 1.6 & 0.85 & .74 \\
\hline 8. Sensación de inestabilidad. & 1.8 & 0.95 & .74 \\
\hline 9. Con miedo o aterrorizado/a. & 1.7 & 0.90 & .77 \\
\hline 10. Nervioso/a. & 2.4 & 0.94 & .77 \\
\hline 11. Sensación de ahogo. & 1.4 & 0.77 & .72 \\
\hline 12. Temblor de manos. & 1.3 & 0.64 & .66 \\
\hline 13. Inquieto/a, tembloroso/a. & 1.6 & 0.80 & .74 \\
\hline 14. Miedo a perder el control. & 1.6 & 0.92 & .77 \\
\hline 15. Dificultad para respirar. & 1.3 & 0.65 & .71 \\
\hline 16. Miedo a morir. & 1.4 & 0.81 & .63 \\
\hline 17. Asustado/a. & 1.7 & 0.90 & .75 \\
\hline $\begin{array}{l}\text { 18. Indigestión o malestar } \\
\text { estomacal. }\end{array}$ & 1.9 & 0.94 & .51 \\
\hline $\begin{array}{l}\text { 19. Sensación de desvanecimiento } \\
\text { o desmayos. }\end{array}$ & 1.1 & 0.32 & .52 \\
\hline 20. Ruborizarse, sonrojamiento. & 1.3 & 0.63 & .50 \\
\hline $\begin{array}{l}\text { 21. Sudoración (no producida } \\
\text { por calor). }\end{array}$ & 1.4 & 0.69 & .55 \\
\hline
\end{tabular}

Nota. $M=$ media; $D E=$ desviación estándar.

En cuanto a las cargas estandarizadas correspondientes al modelo unidimensional obtenidas a partir de los distintos métodos de estimación, los valores oscilaron entre .47 y .78 para RULS, entre .40 y .68 para RML, entre .46 y .85 para DWLS, y entre .72 y .97 para RGLS (véase Tabla 3 ), de manera que los valores de las cargas estandarizadas resultaron adecuados (mayores a .40), independientemente del método de estimación utilizado. Como se puede observar en la Tabla 3, los valores más 
bajos se obtuvieron a partir del método RML, seguido del RULS y el DWLS, y los valores más elevados se obtuvieron mediante el RLGS. En definitiva, las cargas estandarizadas de los reactivos aportan evidencia sobre de la validez de constructo del BAI.

Tabla 3.

Cargas estandarizadas de los reactivos del BAI obtenidas a partir de distintos métodos de estimación

\begin{tabular}{lcccc}
\hline \multicolumn{1}{c}{ Ítem } & RULS & RML & DWLS & RGLS \\
\hline 1. Hormigueo o entumecimiento. & .49 & .41 & .48 & .72 \\
2. Sensación de calor intenso. & .49 & .41 & .48 & .79 \\
3. Debilidad en las piernas. & .58 & .48 & .56 & .82 \\
4. Incapacidad para relajarse. & .68 & .59 & .70 & .88 \\
5. Miedo a que pase lo peor. & .73 & .64 & .78 & .94 \\
6. Mareos o vértigos. & .67 & .57 & .65 & .90 \\
7. Palpitaciones o taquicardia. & .74 & .63 & .72 & .94 \\
8. Sensación de inestabilidad. & .75 & .65 & .75 & .93 \\
9. Con miedo o aterrorizado/a. & .77 & .68 & .85 & .97 \\
10. Nervioso/a. & .78 & .68 & .81 & .96 \\
11. Sensación de ahogo. & .71 & .61 & .73 & .95 \\
12. Temblor de manos. & .65 & .56 & .65 & .89 \\
13. Inquieto/a, tembloroso/a. & .74 & .64 & .74 & .95 \\
14. Miedo a perder el control. & .78 & .68 & .79 & .95 \\
15. Dificultad para respirar. & .70 & .60 & .73 & .96 \\
16. Miedo a morir. & .63 & .54 & .67 & .92 \\
17. Asustado/a. & .75 & .65 & .82 & .97 \\
18. Indigestión o malestar & .50 & .42 & .48 & .70 \\
$\quad$ estomacal. & & & & \\
19. Sensación de desvanecimiento & .51 & .43 & .49 & .78 \\
$\quad$ o desmayos. & .47 & .40 & .46 & .77 \\
20. Ruborizarse, sonrojamiento. & .52 & .44 & .51 & .87 \\
21. Sudoración (no producida & .57 & & \\
$\quad$ por calor). & & & & \\
\hline
\end{tabular}

Por otra parte, para estimar la fiabilidad del BAI, primero se evaluó la función del cálculo del alfa a través del examen de los índices de bondad de ajuste del modelo tau-equivalente. Puntualmente, los valores elevados de chi cuadrado y en el RMSEA resultaron no aceptables, de manera que el cálculo del alfa permitiría estimar la mayor fiabilidad posible en ausencia del cumplimiento del supuesto de tau-equivalencia (véase Tabla 4).

De este modo, con respecto a la fiabilidad, los valores obtenidos a partir de los distintos índices calculados resultaron aceptables en todos los casos, de manera que, en conclusión, también fue posible obtener evidencia sobre de la fiabilidad del BAI (véase Tabla 5).
Tabla 4.

Índices de bondad de ajuste del modelo tau-equivalente a partir de distintos métodos de estimación

\begin{tabular}{lccccc}
\hline Método & $x^{2}$ & GL & NNFI & CFI & RMSEA (IC 90 \%) \\
\hline RULS & 1423.272 & 208 & .93 & .93 & $.09(.08-.09)$ \\
RML & 12267.743 & 208 & .94 & .94 & $.08(.07-.09)$ \\
DWLS & 1439.865 & 189 & .96 & .95 & $.09(.08-.09)$ \\
RGLS & 204.817 & 108 & .97 & .97 & $.04(.03-.04)$ \\
\hline
\end{tabular}

Tabla 5.

Coeficientes de consistencia interna del BAI

\begin{tabular}{cc}
\hline Coeficiente & Valor \\
\hline Alfa & $.94(.93-.95)$ \\
Omega & .95 \\
GLB algebraico & .97 \\
GLB factorial & .96 \\
Beta & .86 \\
H & .91 \\
Theta & .88 \\
\hline
\end{tabular}

\section{Discusión}

En la presente investigación se propuso obtener evidencias acerca de la validez estructural y la consistencia interna de la versión adaptada a población de Buenos Aires del BAI, mediante el uso de distintos métodos.

Así, en primer lugar, se estimó la validez estructural a partir del examen de los modelos de uno, dos y cuatro factores, para lo cual se realizaron estimaciones desde los métodos cuya adecuación para datos ordinales fuera corroborada empíricamente: RULS, DWLS y RML (Bandalos, 2014; Holgado-Tello et al., 2018; Kogar \& Yilmaz-Kogar, 2015; Li, 2016a; Li, 2016b; Nalbantoğlu-Y1lmaz, 2019). Adicionalmente, se utilizó el método RGLS, del cual no se había hallado estudios empíricos en datos ordinales, y se obtuvieron valores de ajuste aceptables para el modelo unidimensional independientemente del método de estimación utilizado. Por lo tanto, el modelo resultó estable a pesar de las diferencias que pudieran existir en función de la posible influencia del método de estimación (Kogar \& Yilmaz-Kogar, 2015). Estos resultados son concordantes con estudios previos respecto a la unidimensionalidad del BAI (Sanz et al., 2012; Sanz \& Navarro, 2003; Vizioli \& Pagano, 2020).

En el caso de los otros modelos, se observó que los índices de bondad de ajuste podían ser inaceptables de acuerdo 
con el método de estimación. Asimismo, se encontró que, con cada método de estimación, los índices de bondad de ajuste del modelo unidimensional resultaron mejores que en los otros dos modelos.

Con respecto a la fiabilidad, los distintos métodos de estimación utilizados para el AFC evidenciaron la no adecuación del modelo tau-equivalente - modelo que tiene la característica de restringir las cargas factoriales (Dunn et al., 2014) - . Al no cumplirse este supuesto, se asumió que el cálculo del alfa ordinal funcionaría para estimar el mayor límite inferior (Cronbach, 1951; Trizano-Hermosilla \& Alvarado, 2016). Así, el valor obtenido fue de .94 - es decir, muy bueno-; un alfa similar al de .92 de Beck et al. (1988), al de 94 de Rodríguez Reynaldo et al. (2001), al de .94 de Liang et al. (2018), y al de .91 de Padrós Blázquez et al. (2020). De hecho, el alfa obtenido fue relativamente superior al alfa de .88 reportado por Sanz y Navarro (2003), al de .83 de Robles et al. (2001), al de .89 de Guillén DíazBarriga y González-Celis Rangel (2019), y al de .90 de Sanz et al. (2012).

De igual manera, se calcularon distintos índices de fiabilidad adecuados para modelos congenéricos; una decisión que tuvo fundamento en la diversidad de recomendaciones acerca de índices de fiabilidad. A partir de ello, se obtuvo un omega ordinal de .95 , con lo cual se obtuvieron valores similares a los obtenidos por Vizioli y Pagano (2020), quienes hallaron un alfa ordinal de .93 y un omega ordinal de .95 . Como era de esperarse, los valores de omega fueron ligeramente más elevados que los obtenidos mediante el coeficiente alfa (Revelle \& Zinbarg, 2009). Al respecto, es preciso mencionar que se estimó solo un omega, dado que, al ser el modelo unidimensional, el omega total y el jerárquico son equivalentes (TrizanoHermosilla \& Alvarado, 2016).

Asimismo, se calcularon índices de fiabilidad que resultan novedosos en estudios psicométricos del BAI, como lo fueron el GLB algebraico, el GLB factorial, el $\mathrm{H}$ y la theta. En todos los casos, se obtuvieron valores considerados aceptables: GLB algebraico $=.97$, GLB factorial $=.96$, beta $=.86 ; \mathrm{H}=.91 \mathrm{y}$ theta $=.88$. En concordancia, se podría a firmar que la versión del BAI adaptada a población de Buenos Aires demuestra ser fiable independientemente del coeficiente calculado.
Finalmente, este trabajo presenta algunas limitaciones que deben tenerse en cuenta, como que la muestra empleada no incluye participantes de regiones distintas del área metropolitana de Buenos Aires, o que no se realizaron análisis de la estabilidad temporal, la validez discriminante o la invarianza factorial por género. Estas cuestiones deberán contemplarse en nuevos estudios para que se recolecten muestras más heterogéneas de distintas regiones, e incluso con métodos de muestreo probabilísticos que briden la posibilidad de extrapolar los resultados obtenidos al total de la población local.

De igual forma, se propone que futuras investigaciones indaguen evidencias de estabilidad temporal del instrumento, así como de la validez discriminante en relación con variables como la sintomatología depresiva. Además, se sugiere analizar la invarianza factorial según el género, dado que esta variable no ha sido muy abordada dentro de la literatura investigativa. No obstante, aunque se entiende que las propiedades psicométricas de un instrumento deben ser puestas a prueba constantemente y con diferentes procedimientos y muestras, se debe reconocer que se trata de un proceso continuo que debe contar con múltiples evidencias que no pueden ser abordadas todas en una única investigación.

En líneas generales, la presente investigación permitió obtener evidencias acerca de la validez estructural y la fiabilidad del BAI a través de distintos métodos, pues los resultados obtenidos dan cuenta de la solidez de las propiedades psicométricas del instrumento. De esta manera, el instrumento se presenta como una alternativa importante para su uso en ámbitos como el investigativo o el clínico, dada la brevedad de su administración (Bardhoshi et al., 2016), y una característica importante del BaI radica en que, a diferencia de otros instrumentos que miden la ansiedad, evalúa síntomas que no se comparten con la depresión, lo que facilita la discriminación entre ambos cuadros (Sanz, 2014). Finalmente, se espera que la presente investigación ayude a promover la utilización de distintos métodos de estimación en AFC, así como el cálculo de distintos coeficientes de fiabilidad, con el fin de asegurar la independencia de las propiedades psicométricas de los instrumentos frente a los cálculos realizados. 


\section{Referencias}

Adhikari, C. (2019). Application and validation of the beck anxiety inventory among Nepalese School Adolescents. Journal of Health and Allied Sciences, 9(1), 51-58. https://www.jhas.org.np/jhas/index.php/jhas/article/ view/10/9

Armor, D. J. (1973). Theta reliability and factor scaling. Sociological Methodology, 5, 17-50. https://doi. org/10.2307/270831

Bandalos, D. L. (2014). Relative performance of categorical diagonally weighted least squares and robust maximum likelihood estimation. Structural Equation Modeling: a Multidisciplinary Journal, 21(1), 102-116. https://doi.or g/10.1080/10705511.2014.859510

Bardhoshi, G., Duncan, K., \& Erford, B. T. (2016). Psychometric meta-analysis of the English version of the Beck Anxiety Inventory. Journal of Counseling \& Development, 94(3), 356-373. https://doi.org/10.1002/ jcad. 12090

Beck, A. T., \& Steer, R. A. (1993). Beck Anxiety Inventory manual. Psychological Corporation.

Beck, A. T., Epstein, N., Brown, G., \& Steer, R. A. (1988). An inventory for measuring clinical anxiety: Psychometric properties. Journal of Consulting and Clinical Psychology, 56(6), 893-897. https://doi. org/10.1037/0022-006X.56.6.893

Benuto, L. T., Zimmermann, M., Gonzalez, F. R., \& Corral Rodríguez, A. (2020). A confirmatory factor analysis of the beck anxiety inventory in Latinx primary care patients. International Journal of Mental Health, 49(4), 361-381. https://doi.org/10.1080/00207411.2020.1812833

Bernaards, C. A., \& Jennrich, R. I. (2005). Gradient projection algorithms and software for arbitrary rotation criteria in factor analysis. Educational and Psychological Measurement, 65, 676-696. https://doi. org/10.1177/0013164404272507
Brown, T. A. (2006). Confirmatory factor analysis for applied research (2. ${ }^{\mathrm{a}}$ ed.). Guilford publications.

Browne, M. W., \& Cudeck, R. (1993). Alternative ways of assessing model fit. En K. A. Bollen \& J. S. Long (Eds.), Testing structural equation models (pp. 136-162). Sage.

Chapa, O. (2021). An examination of the Beck Anxiety Inventory structure and psychometric properties: A study of American employees. The International Journal of Human Resource Management, 1-22. https://doi.org/10 $.1080 / 09585192.2020 .1863246$

Clark, J. M., Marszalek, J. M., Bennett, K. K., Harry, K. M., Howarter, A. D., Eways, K. R., \& Reed, K. S. (2016). Comparison of factor structure models for the Beck Anxiety Inventory among cardiac rehabilitation patients. Journal of Psychosomatic Research, 89, 91-97. https:// doi.org/10.1016/j.jpsychores.2016.08.007

Coenders, G., Satorra, A., \& Saris, W. E. (1997). Alternative approaches to structural modeling of ordinal data: A Monte Carlo study. Structural Equation Modeling: A Multidisciplinary Journal, 4(4), 261-282. https://doi. org/10.1080/10705519709540077

Cronbach, L. J. (1951). Coefficient alpha and the internal structure of tests. Psychometrika, 16(3), 297-334. https:// doi.org/10.1007/BF02310555

Cupani, M. (2012). Análisis de Ecuaciones Estructurales: conceptos, etapas de desarrollo y un ejemplo de aplicación. Revista tesis, 2, 186-199. https://revistas.unc.edu. ar/index.php/tesis/article/download/2884/2750/0

Datani, S., Ritchie, H., \& Roser, M. (2021). Mental Health. Our World in Data. https://ourworldindata.org/ mental-health

DiStefano, C., McDaniel, H. L., Zhang, L., Shi, D., \& Jiang, Z. (2019). Fitting large factor analysis models with ordinal data.Educational and Psychological Measurement, 79(3), 417-436. https://doi.org/10.1177/0013164418818242 
Domínguez-Lara, S. A. (2012). Propuesta para el cálculo del Alfa Ordinal y Theta de Armor. Revista de investigación en Psicología, 15(1), 213-217. https://doi.org/10.15381/ rinvp.v15i1.3684

Domínguez-Lara, S. A. (2016). Evaluación de la fiabilidad del constructo mediante el Coeficiente $\mathrm{H}$ : breve revisión conceptual y aplicaciones. Psychologia. Avances de la disciplina, 10(2), 87-94. https://doi. org/10.21500/19002386.2134

Dominguez-Lara, S. (2018). Fiabilidad y alfa ordinal. Actas Urológicas Españolas, 42(2), 140-141. https://doi.org/10.1016/j.acuro.2017.07.002

Domínguez-Lara, S. A., \& Merino-Soto, C. (2015). ¿Por qué es importante reportar los intervalos de confianza del coeficiente alfa de Cronbach? Revista Latinoamericana de Ciencias Sociales, Niñez y Juventud, 13(2), 1326-1328. http://ns520666.ip-158-69-118.net/rlcsnj/index.php/ Revista-Latinoamericana/article/download/2030/629

Dunn, T. J., Baguley, T., \& Brunsden, V. (2014). From alpha to omega: A practical solution to the pervasive problem of internal consistency estimation. British Journal of Psychology, 105(3), 399-412. https://doi.org/10.1111/bjop.12046

Elosua, P., \& Zumbo, B. (2008). Coeficientes de fiabilidad para escalas de respuesta categórica ordenada. Psicothema, 20(4), 896-901. http://www.psicothema. com/pdf/3572.pdf

Flora, D. B., \& Curran, P. J. (2004). An empirical evaluation of alternative methods of estimation for confirmatory factor analysis with ordinal data. Psychological Methods, 9(4), 466-491. https://psycnet.apa.org/ doi/10.1037/1082-989X.9.4.466

Fox, J., Bouchet-Valat, M., Andronic, L., Ash, M., Boye, T., Calza, S., Chang, A., Gegzna, V., Grosjean, P., Heiberger, R., Karimi Pour, K., Kerns, G. J., Lancelot, R., Lesnoff, M., Ligges, U., Messad, S., Maechler, M., Muenchen, R., Murdoch, D., \& Wright, M. (2019). Rcmdr: $R$ Commander. $R$ package version 2.5-2. https://cran.r-project.org/web/packages/Rcmdr/index.html
Freiberg-Hoffmann, A., Abal, F., \& Fernández-Liporace, M. (2020). Cuestionario Honey-Alonso de Estilos de Aprendizaje: Nuevas evidencias psicométricas en población argentina. Acta Colombiana de Psicología, 23(2), 328-338. https://doi.org/10.14718/ACP.2020.23.2.13

Gadermann, A. M., Guhn, M., \& Zumbo, B. D. (2012). Estimating ordinal reliability for Likert-type and ordinal item response data: A conceptual, empirical, and practical guide. Practical Assessment, Research, and Evaluation, 17(3), 1-13. https://doi.org/10.7275/n560-j767

Guillén Díaz-Barriga, C., \& González-Celis Rangel, A. L. (2019). Propiedades psicométricas del Inventario de Ansiedad de Beck en adultos asmáticos mexicanos. Psicología y Salud, 29(1), 5-16. https://psicologiaysalud. uv.mx/index.php/psicysalud/article/view/2563/4471

Hancock, G. R., \& Mueller, R. O. (2001). Rethinking construct reliability within latent variable systems. En R. Cudeck, S. du Toit \& D. Soerbom (Eds.), Structural equation modeling: Present and future-A festschrift in honor of Karl Jöreskog (pp. 195-216). Scientific Software International.

Henseler, J., Ringle, C. M., \& Sinkovics, R. R. (2009). The use of partial least squares path modeling in international marketing. En R. R. Sinkovics \& P. N. Ghauri (Eds.), Advances in International Marketing (vol. 20, pp. 277-320). Emerald. https://doi.org/10.1108/ S1474-7979(2009)0000020014

Holgado-Tello, F. P., Morata-Ramírez, M. Á., \& BarberoGarcía, M. I. (2018). Confirmatory Factor Analysis of Ordinal Variables: A Simulation Study Comparing the Main Estimation Methods. Avances en Psicología Latinoamericana, 36(3), 601-617. http://dx.doi. org/10.12804/revistas.urosario.edu.co/apl/a.4932

Hoyle, R. H. (2012). Introduction and overview. En Hoyle, R. H. (Ed.), Handbook of structural equation modeling. The Guilford Press.

Jackson, P. H., \& Agunwamba, C. C. (1977). Lower bounds for the reliability of the total score on a test composed 
of non-homogeneous items: I: Algebraic lower bounds. Psychometrika, 42(4), 567-578. https://doi.org/10.1007/ BF02295979

Jöreskog, K. G., \& Sörbom , D. (1996). LISREL 8: User's reference guide. Scientific Software International.

Kline, R. B. (2011). Principles and practice of structural equation modeling (3. ${ }^{\mathrm{a}}$ ed.). Guilford Press.

Kogar, H., \& Yilmaz-Kogar, E. (2015). Comparison of different estimation methods for categorical and ordinal data in confirmatory factor analysis. Journal of Measurement and Evaluation in Education and Psychology, 6(2), 351364. https://doi.org/10.21031/epod.94857

Lee, K., Kim, D., \& Cho, Y. (2018). Exploratory Factor Analysis of the Beck Anxiety Inventory and the Beck Depression Inventory-II in a Psychiatric Outpatient Population. Journal of Korean Medical Science, 33(16), e128. https://doi.org/10.3346/jkms.2018.33.e128

Lei, P. W., \& Wu, Q. (2012). Estimation in Structural Equation Modeling. En Hoyle, R. H. (Ed.), Handbook of structural equation modeling. The Guilford Press.

Li, C. H. (2016a). Confirmatory factor analysis with ordinal data: Comparing robust maximum likelihood and diagonally weighted least squares. Behavior Research Methods, 48(3), 936-949. https://doi.org/10.3758/ s13428-015-0619-7

Li, C. H. (2016b). The performance of ML, DWLS, and ULS estimation with robust corrections in structural equation models with ordinal variables. Psychological Methods, 21(3), 369-387. https://doi.org/10.1037/met0000093

Liang, Y., Wang, L., \& Zhu, J. (2018). Factor structure and psychometric properties of Chinese version of Beck Anxiety Inventory in Chinese doctors. Journal of Health Psychology, 23(5), 657-666. https://doi. org/10.1177/1359105316658971

Magán, I., Sanz, J., \& García-Vera, M. P. (2008). Psychometric properties of a Spanish version of the Beck
Anxiety Inventory (BAI) in general population. The Spanish Journal of Psychology, 11(2), 626-640. https:// doi.org/10.1017/S1138741600004637

McDonald, R. P. (1999). Test theory: A unified treatment. Erlbaum.

McNeish, D. (2018). Thanks coefficient alpha, we'll take it from here. Psychological Methods, 23(3), 412-433. https://psycnet.apa.org/doi/10.1037/met0000144

Montero, I., \& León, O. G. (2002). Clasificación y descripción de las metodologías de investigación en Psicología. International Journal of Clinical and Health Psychology, 2(3), 503-508. http://www.aepc.es/ijchp/articulos_pdf/ij chp-53.pdf

Morata-Ramírez, M., Holgado-Tello, F. P., Barbero-García, I., \& Mendez, G. (2015). Análisis factorial confirmatorio: recomendaciones sobre mínimos cuadrados no ponderados en función del error Tipo I de Ji-Cuadrado y RMSEA. Acción Psicológica, 12(1), 79-90. http://scielo. isciii.es/pdf/acp/v12n1/articulo8.pdf

Moltner, A., \& Revelle, W. (2015). Find the Greatest Lower Bound to Reliability. http://personality-project.org/r/ psych/help/glb.algebraic.html

Nalbantoğlu-Yılmaz, F. (2019). Comparision of Different Estimation Methods Used in Confirmatory Factor Analyses in Non-Normal Data: A Monte Carlo Study. International Online Journal of Educational Sciences, 11(4), 131-140. http://dx.doi.org/10.15345/ iojes.2019.04.010

Nunnally, J., \& Bernstein, I. (1994). Psychometric Theory (3. ${ }^{\mathrm{a}}$ ed.). MacGraw-Hill.

Oliden, P. E., \& Zumbo, B. D. (2008). Coeficientes de fiabilidad para escalas de respuesta categórica ordenada. Psicothema, 20(4), 896-901. http://www.psicothema. com/pdf/3572.pdf

Padrós Blázquez, F., Montoya Pérez, K. S., Bravo Calderón, M. A., \& Martínez Medina, M. P. (2020). Propiedades 
psicométricas del Inventario de Ansiedad de Beck (BAI, Beck Anxiety Inventory) en población general de México. Ansiedad y Estrés, 26(2-3), 181-187. https://doi. org/10.1016/j.anyes.2020.08.002

Palmer, G. A., Happe, M. C., Paxson, J. M., Jurek, B. K., \& Olson, S. A. (2016). Dimensionality of the Beck Anxiety Inventory in a polytrauma sample of $\mathrm{OEF} / \mathrm{OIF} / \mathrm{OND}$ veterans: Findings from exploratory and confirmatory factor analysis. Military Psychology, 28(5), 296-305. https://doi.org/10.1037/mil0000117

Piotrowski, C. (2018). The status of the Beck inventories (BDI, BAI) in psychology training and practice: $\mathrm{A}$ major shift in clinical acceptance. Journal of Applied Biobehavioral Research, 23(3), e12112. https://doi. org/10.1111/jabr.12112

Raykov, T. (1998). Coefficient alpha and composite reliability with interrelated nonhomogeneous items. Applied Psychological Measurement, 22(4), 375-385. https://doi. org/10.1177/014662169802200407

Raykov, T., \& Marcoulides, G. A. (2006). A first course in structural equation modeling (2. ${ }^{\mathrm{a}}$ ed.). Lawrence Erlbaum Associates Publishers.

Raykov, T., \& Marcoulides, G. A. (2019). Thanks coefficient alpha, we still need you! Educational and Psychological Measurement, 79(1), 200-210. https://doi. org/10.1177/0013164417725127

Revelle, W. (1979). Hierarchical cluster analysis and the internal structure of tests. Multivariate Behavioral Research, 14(1), 57-74. https://doi.org/10.1207/ s15327906mbr1401_4

Revelle, W. (2018). Psych: Procedures for psychological, psychometric, and per-sonality research. $R$ package version 1.8.12. https://cran.rproject.org/web/packages/ psych/index.html

Revelle, W., \& Zinbarg, R. E. (2009). Coefficients alpha, beta, omega, and the glb: Comments on Sijtsma.
Psychometrika, 74(1), 145-154. https://doi.org/10.1007/ s11336-008-9102-z

Robles, R., Varela, R., Jurado, S., \& Páez, F. (2001). Versión mexicana del Inventario de Ansiedad de Beck: propiedades psicométricas. Revista Mexicana de Psicología, 18(2), 211-218.

Rodríguez Reynaldo, M., Martínez Lugo, M., \& Rodríguez Gómez, J. R. (2001). Estudio de las características psicométricas del Inventario de Ansiedad Beck (en castellano) en una muestra de envejecidos puertorriqueños. Revista Española de Geriatría y Gerontología, 36(6), 353-360. https://doi.org/10.1016/S0211-139X(01)74755-5

Rosseel, Y. (2012). Iavaan: An R Package for Structural Equation Modeling. Journal of Statistical Software, 48(2), 1-36. http://www.jstatsoft.org/v48/i02/

Sanz, J. (2014). Recomendaciones para la utilización de la adaptación española del Inventario de Ansiedad de Beck (BAI) en la práctica clínica. Clínica y Salud, 25(2014), 3948. https://scielo.isciii.es/pdf/clinsa/v25n1/original4.pdf

Sanz, J., García-Vera, M. P., \& Fortún, M. (2012). El Inventario de ansiedad de Beck (BAI): Propiedades psicométricas de la versión española en pacientes con trastornos psicológicos. Psicología Conductual, 20(3), 563584. https://www.behavioralpsycho.com/wp-content/ uploads/2019/08/05.Sanz_20-3oa-1.pdf

Sanz, J., \& Navarro, M. E. (2003). Propiedades psicométricas de una versión española del inventario de ansiedad de Beck (BAI) en estudiantes. Ansiedad y Estrés, 9(1), 59-84. https://pesquisa.bvsalud.org/portal/resource/pt/ ibc- 22883

Savalei, V., \& Rhemtulla, M. (2013). The performance of robust test statistics with categorical data. British Journal of Mathematical and Statistical Psychology, 66(2), 201223. https://doi.org/10.1111/j.2044-8317.2012.02049.x

Schumacker, R., \& Lomax, R. (2016). A beginner's guide to structural equation modeling (4. ${ }^{\mathrm{a}}$ ed.). Routledge. 
Sijtsma, K. (2009). On the use, the misuse, and the very limited usefulness of Cronbach's alpha. Psychometrika, 74(1), 107-120. https://doi.org/10.1007/s11336-008-9101-0

Trizano-Hermosilla, I., \& Alvarado, J. M. (2016). Best alternatives to Cronbach's alpha reliability in realistic conditions: congeneric and asymmetrical measurements. Frontiers in Psychology, 7, Article 769. https://doi. org/10.3389/fpsyg.2016.00769

Viladrich, C., Angulo-Brunet, A., \& Doval, E. (2017). Un viaje alrededor de alfa y omega para estimar la fiabilidad de consistencia interna. Anales de Psicología, 33(3), 755-782. https://revistas.um.es/analesps/article/view/ analesps.33.3.268401/215531

Vizioli, N., \& Pagano, A. (2020). Adaptación del Inventario de Ansiedad de Beck en población de Buenos Aires. Interacciones, 6(3), e171. https://doi.org/10.24016/2020. v6n3.171
World Medical Association. (2013). Declaration of Helsinki. Ethical principles for medical research involving human subjects. JAMA Network, 310(20), 2191-2194. https:// doi.org/10.1001/jama.2013.281053

Yang-Wallentin, F., Jöreskog, K. G., \& Luo, H. (2010). Confirmatory factor analysis of ordinal variables with misspecified models. Structural Equation Modeling, 17(3), 392-423. https://psycnet.apa.org/doi/10.1080/107 05511.2010 .489003

Zumbo, B. D., Gadermann, A. M., \& Zeisser, C. (2007). Ordinal versions of coefficients alpha and theta for Likert rating scales. Journal of Modern Applied Statistical Methods, 6(1), Article 4. https://doi.org/10.22237/ jmasm/1177992180 